\title{
SOLUCCÃO DE EQUAÇÃO DE BALANÇO POPULACIONAL VIA TÉCNICA DA TRANSFORMADA DE LAPLACE.
}

\author{
C. H. RODRIGUES DE MOURA ${ }^{1}$, C. DA SILVA BATISTA ${ }^{1}$ \\ ${ }^{1}$ Universidade Federal do Pará, Faculdade de Engenharia Química \\ E-mail para contato: carlos.moura@itec.ufpa.br
}

\begin{abstract}
RESUMO - A descrição de uma variedade de processos envolvendo a formação de sistemas particulados requer um entendimento das equações de balanço populacional (PBE). Essas equações buscam prever a evolução da distribuição de uma ou mais propriedades que caracterizam o indivíduo, partículas ou entidades, e a PBE dinâmica é em essência uma equação de balanço de número para descrever essa evolução. O desenvolvimento de métodos para resolver a equação de $\mathrm{PB}$ tem sido uma área de investigação ativa ao longo das duas últimas décadas. Para esse trabalho, propõe-se usar a Técnica da Transformada de Laplace na solução de problemas de balanço populacional (PB) com formulações hiperbólicas e não lineares na forma integro-diferencial parcial o qual é raramente tratada analiticamente. A partir da sua resolução, é possível estimar a função densidade de tamanho de partículas, e assim prever o comportamento dinâmico do sistema físico.
\end{abstract}

\section{INTRODUÇÃO}

Com vistas de implantar um projeto para uma nova planta química para região Amazônica, é imperioso compreender o processo de precipitação do tri - hidróxido de alumínio $(\mathrm{Al}(\mathrm{OH}) 3)$ no Processo Bayer, já que o mesmo é responsável pela morfologia, granulometria, qualidade da alumina e consequentemente é responsável direto pela qualidade do alumínio primário produzido nas reduções.

A precipitação acontece através de várias etapas, sendo resultado de vários mecanismos, dentre eles a nucleação, cementação, agregação e quebra, conforme mostra a figura 1. Como o processo é rápido, com mistura em várias escalas, desempenha um importante papel na determinação da distribuição de tamanho do cristal final (CSD) e na morfologia dos cristais (MARCHISIO et al., 2002).

Figura 1 - Precipitação de partículas: nucleação, cementação, quebra e aglomeração. 

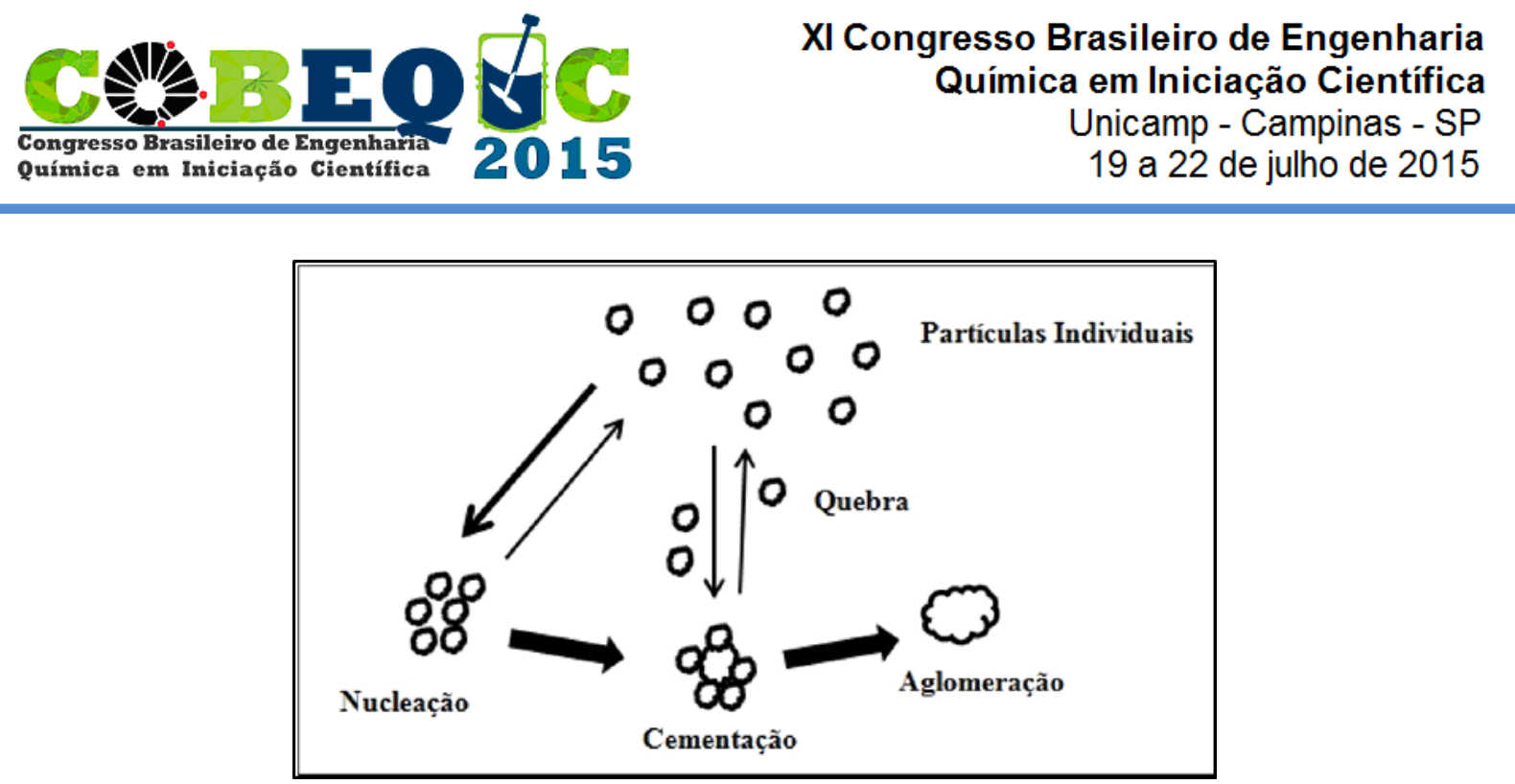

O estabelecimento de um modelo de Balanço Populacional (PB) em um sistema de precipitação é geralmente simples. Nas formulações matemáticas são estabelecidos os termos para descrever a aglomeração de cristais, o processo onde os cristais colidem e aderem para formar uma entidade maior, crescimento de cristais pela deposição de soluto da solução, e nucleação. No entanto, a resolução da equação de PB pode ser difícil, especialmente quando a aglomeração está presente. Soluções analíticas existem apenas para casos simples, por exemplo, onde qualquer aglomeração ou o crescimento é o único processo cinético envolvido no sistema. $\mathrm{O}$ desenvolvimento de métodos para resolver a equação de PB tem sido uma área de investigação ativa ao longo das últimas duas décadas.

A equação de Balanço Populacional (PBE) dada pela Equação (1) foi proposta por Ilievski (2001) que representa a precipitação com supersaturação constante e núcleo de aglomeração da gibsita (subproduto do processo Bayer em refinaria de alumina) independente do tamanho da partícula.

$$
\frac{\partial n(v, t)}{\partial t}=-G \frac{\partial n(v, t)}{\partial v}+\frac{\beta}{2} \int_{0}^{v} n(\bar{v}, t) n(v-\bar{v}, t) d \bar{v}-\beta n(v, t) \int_{0}^{\infty} n(\bar{v}, t) d \bar{v}
$$

Sujeito as seguintes condições inicial e de contorno:

$$
\begin{aligned}
& n(v, 0)=\frac{N_{0}}{v_{0}} e^{-v / v_{0}} \\
& n(0, t)=0
\end{aligned}
$$

$\mathrm{Na}$ Equação (1) acima, o termo $\mathrm{G}=\mathrm{dv} / \mathrm{dt}$, é a taxa de crescimento de partículas de volume $\mathrm{v}, \mathrm{n}(\mathrm{v}, \mathrm{t})$ é a função número de densidade cristalina, $\beta(v, \tilde{v})$ é o coeficiente de coagulação para partículas de volume $\mathrm{v}$ e $\tilde{v}$. O primeiro termo do lado direito está relacionado à taxa de crescimento de partículas por transferência de material para partículas individuais, o segundo representa o acúmulo de partículas na escala de tamanho $(\mathrm{v}, \mathrm{v}+\mathrm{dv})$ pela colisão de duas partículas $\mathrm{v}-\tilde{v}$ e para formar uma partícula de volume (assumindo conservação de volume durante a coagulação), o terceiro representa a taxa de perda na escala de tamanho de partícula $(\mathrm{v}, \mathrm{v}+\mathrm{dv})$ pela colisão com todas as outras partículas.

\section{METODOLOGIA}


A técnica da Transformada de Laplace é uma ferramenta poderosa na determinação de soluções de equações diferenciais ordinárias com condições iniciais. O operador $L$ é um operador integral (linear) que remove derivadas, transformando EDOs em equações algébricas simples. A transformada de Laplace é definida por uma integral variando de zero a infinito. A seguir algumas particularidades da transformada de Laplace (RAINVILLE, 1964; BALDINO, 1979):

I) Definição da transformada de Laplace: Seja $F(t)$ uma função de $t$ definida para $t>0$. Então, a transformada de Laplace de $\mathrm{F}(\mathrm{t})$ é representada por é definida por:

$$
L\{F(t)\}=f(s)=\int_{0}^{\infty} e^{-s t} F(t) d t
$$

II) Notação: Se uma função de t é indicada em termos de uma letra maiúscula, tal como $F(t), G(t), Y(t)$, etc., a transformada de Laplace da função é denominada pela letra minúscula correspondente, isto é, $\mathrm{f}(\mathrm{s}), \mathrm{g}(\mathrm{s}), \mathrm{y}(\mathrm{s})$, etc. Em outros casos, um til $(\sim)$ pode ser usado para denotar a transformada de Laplace.

III) Condições suficientes para existência da transformada de Laplace: Teorema: Se F(t) é seccionalmente contínua em todo intervalo finito $0 \leq t \leq N$ e de ordem exponencial $\gamma$ para $t>N$, então sua transformada de Laplace $f(s)$ existe para todo $s>\gamma$.

IV) Métodos para encontrar transformadas de Laplace: Há vários métodos disponíveis para determinar transformadas de Laplace, como indicado na seguinte lista: Método direto, Método das séries, Método das equações diferenciais, Método em relação a um parâmetro, Diferenciação em relação a um parâmetro, Métodos diversos, Uso de tabelas.

V) A transformada inversa de Laplace: Se a transformada de Laplace de uma $\mathrm{F}(\mathrm{t})$ é $f(s)$, isto é, se $L\{F(t)\}=f(s)$, então $\mathrm{F}(\mathrm{t})$ é chamada transformada inversa de Laplace de $f(s)$ e escreve-se simbolicamente $F(t)=L^{-1}\{f(s)\}$, onde é chamado operador da transformada inversa de Laplace.

VI) Métodos para encontrar transformadas inversas de Laplace: Método das frações parciais, Método das séries, Método das equações diferenciais, Diferenciação em relação a um parâmetro, Métodos diversos usando os teoremas acima, Uso de tabelas, A fórmula complexa de inversão.

\section{RESULTADOS}

Para resolver o modelo matemático dado pela equação íntegro diferencial parcial proposto por RAMABHADRAN et al. (1976), a técnica da transformada de Laplace foi empregada. Para este caso, considerou-se a taxa de coagulação constante $\left(\beta=\beta_{0}\right)$ e taxa de condensação linear: 


$$
\begin{aligned}
\frac{\partial n(v, t)}{\partial t}+G \frac{\partial[v n(v, t)]}{\partial v}= & D_{a b} \frac{\partial^{2} n(v, t)}{\partial v^{2}}+\frac{\beta}{2} \int_{0}^{v} n(\bar{v}, t) n(v-\bar{v}, t) d \bar{v} \\
& -\beta n(v, t) \int_{0}^{\infty} n(\bar{v}, t) d \bar{v}
\end{aligned}
$$

Com as seguintes condições de contorno e inicial:

$$
\begin{aligned}
& n(0, t)=0 \\
& \frac{\partial n(\mathrm{v}, \mathrm{t})}{\partial v}=0 \\
& n(v, 0)=\frac{N_{0}}{v_{0}} e^{-v / v_{0}}
\end{aligned}
$$

Aplicando a transformada de Laplace em cada termo da Equação (3.1), a fim de remover a variável independente $\mathrm{v}$, obtém-se:

$$
\begin{aligned}
& \mathrm{L}\left[\frac{\partial n(v, t)}{\partial t}\right]=\frac{\partial \bar{n}(\mathrm{~s}, t)}{\partial t} ; \mathrm{L}\left[\frac{\partial[v n(v, t)]}{\partial v}\right]=-s \frac{d \bar{n}(s, t)}{d s} \\
& \mathrm{~L}\left[\frac{\partial^{2} n(v, t)}{\partial v^{2}}\right]=s^{2} \bar{n}(s, t) \\
& \mathrm{L}\left[\int_{0}^{v} n(\bar{v}, t) n(v-\bar{v}, t) d \bar{v}\right]=\bar{n}(s, t)^{2} \\
& \mathrm{~L}[n(v, t)]=\bar{n}(s, t)
\end{aligned}
$$

Portanto, a equação diferencial transformada para este caso, a partir dos resultados das Equações (3.2), juntamente com a condição inicial, dada pela Eq. (3.1.c), também transformada, são escritas como:

$$
\begin{aligned}
& \frac{\partial \bar{n}(\mathrm{~s}, t)}{\partial t}-G s \frac{\partial n(\mathrm{~s}, t)}{\partial s}=D_{a b} s^{2} \bar{n}(s, t)+\frac{\beta_{0}}{2} \bar{n}(s, t)^{2}-\beta M o(t) n(\mathrm{~s}, t) \\
& n(s, 0)=\frac{\left(N_{0} / v_{0}\right)}{s+\left(1 / v_{0}\right)}
\end{aligned}
$$

O momento de ordem zero $M o(t)$ que aparece na Eq. (3.3) é obtido a partir da sua definição usual sendo dado por (RAMABHADRAN et al., 1976):

$$
M o(t)=\int_{0}^{\infty} n(v, t) d v=\frac{2 N_{0}}{2+\beta_{0} N_{0} t}
$$

Rearranjando, 


$$
\frac{\partial \bar{n}(\mathrm{~s}, t)}{\partial t}-G s \frac{\partial n(\mathrm{~s}, t)}{\partial s}=\left(D_{a b} s^{2}-\beta M o\right) \bar{n}(s, t)+\frac{\beta_{0}}{2} \bar{n}(s, t)^{2}
$$

Resolvendo a Equação (3.6) através do método das características:

$$
\begin{aligned}
& \frac{d s}{d \eta}=\frac{d s}{d t}=-G s \\
& s_{0}=s \exp (G t) \\
& \frac{\partial \bar{n}(\mathrm{~s}, t)}{\partial t}=\left(D_{a b} s^{2}-\beta M o\right) \bar{n}(s, t)+\frac{\beta_{0}}{2} \bar{n}(s, t)^{2}
\end{aligned}
$$

Substituindo a Eq. (3.9.b) na Eq. (3.4), obtém-se a condição inicial:

$$
\overline{\mathrm{n}}(s, 0)=\frac{\left(N_{0} / v_{0}\right)}{s \exp (G t)+\left(1 / v_{0}\right)}
$$

Resolvendo a Eq. (3.9.c) pelo teorema de Bernoulli e usando a distribuição inicial dada pela Eq. (3.9.d), resulta:

$$
\bar{n}(s, t)=\frac{2 \beta_{0} N_{0}^{2}}{2 \beta_{0} N_{0}^{2} C e^{-D_{a b} s^{2} t}\left(2+\beta_{0} N_{0} t\right)^{2}-e^{\frac{-2 D_{a b} s^{2}}{\beta_{0} N_{0}}-D_{a b} s^{2} t}\left(2+\beta_{0} N_{0} t\right)(\varphi)}
$$

Onde,

$$
\varphi=-\beta_{0} e^{\frac{-D_{a b} s^{2}\left(2+\beta_{0} N_{0} t\right)}{\beta_{0} N_{0}}} N_{0}+D_{a b} s^{2}\left(2+\beta_{0} N_{0} t\right) \operatorname{Ei}\left[\frac{D_{a b} s^{2}\left(2+\beta_{0} N_{0} t\right)}{\beta_{0} N_{0}}\right]
$$

Então em $t=0$ têm-se

$$
C=\frac{2 \beta_{0} N_{0}\left(v_{0} s e^{g t}+1\right)+2 e^{\frac{-2 D_{a b} s^{2}}{\beta_{0} N_{0}}}\left(-\beta_{0} e^{\frac{-2 D_{a b} s^{2}}{\beta_{0} N_{0}}} N_{0}+2 D_{a b} s^{2} \operatorname{Ei}\left[\frac{2 D_{a b} s^{2}}{\beta_{0} N_{0}}\right]\right)}{8 \beta_{0} N_{0}^{2}}
$$

Substituindo a Equação (3.7.g) na Equação (3.7.e), chega-se na Equação (3.8) abaixo: 


$$
\begin{aligned}
n(s, t)=\left(8 \beta_{0} e^{-D_{a b} s^{2} t} N_{0}{ }^{2}\right) & \left(2+N_{0} \beta_{0} t\right)\left(2 \beta _ { 0 } N _ { 0 } \left(2+2 e^{-\frac{D_{a b} b^{2}\left(4+N_{0} \beta_{0} t\right)}{\beta_{0} N_{0}}}+N_{0} \beta_{0} t-e^{\frac{-4 D_{a b} s^{2}}{\beta_{0} N_{0}}}\left(2+\beta_{0} N_{0} t\right)+\right.\right. \\
& \left.e^{g t} \mathrm{~s}\left(2+\beta_{0} N_{0} t\right) v_{0}\right)+4 D_{a b} e^{-\frac{2 D_{a b} s^{2}}{\beta_{0} N_{0}}} s^{2}\left(2+\beta_{0} N_{0} t\right)\left(E i\left[\frac{2 D_{a b} s^{2}}{\beta_{0} N_{0}}\right]+\right. \\
& \left.-E i\left[\frac{D_{a b} s^{2}\left(2+N_{0} \beta_{0} t\right)}{\beta_{0} N_{0}}\right]\right)
\end{aligned}
$$

\section{CONCLUSÃO}

Para a solução dos modelos matemático, aplicou-se a técnica da transformada de Laplace nas equações diferenciais parciais em relação ao volume com o coeficiente de coagulação constante $\left(\beta=\beta_{0}\right)$. Além do método das características, a solução de Bernoulli foi utilizada para a solução do modelo de balanço populacional. O próximo passo será desenvolver códigos computacionais em linguagem Fortran 90/95 para estimar a função densidade de partículas, $\mathrm{n}(\mathrm{v}, \mathrm{t})$. Resultados numéricos serão obtidos e comparados com os disponíveis na literatura para sistemas particulados, o que vai nos permitir uma avaliação crítica da presente metodologia de solução.

\section{REFERÊNCIAS BIBLIOGRÁFICAS}

BALDINO, R. R., 1979, Transformada de Laplace, Ed. McGraw-Hill do Brasil, LTDA.

BATISTA, C. S., 2011, Solução de equações de balanço populacional usando a técnica da transformada de Laplace e filtro de partículas, Tese - UFPA. Instituto de Tecnologia.

ILIEVSKI, D., 1991, Modelling $\mathrm{Al}(\mathrm{OH})_{3}$ Agglomeration during Butch and Continuos Precipitation in Supersaturated Caustic Aluminate Solutions, Thesis Doctor, University of Queensland, July.

ILIEVSKI, D., 2001, "Development and Application of a Constant Supersaturation, SemiBatch Crystalliser for Investigating Gibbsite Agglomeration", Journal of Crystal Growth, vol. 233, pp 846-862.

MARCHISIO, D. L., BARRESI, A. A., GARBERO, M., 2002, "Nucleation, Growth, and Agglomeration in Barium Sulfate Turbulent Precipitation”, AIChE Journal, Vol. 48, No. 9, pp. $2039-2050$.

RAINVILLE, E. D. 1964, Elementary Differential Equations, Macmillan Company, Third Edition, New York.

RAMABHADRAN T.E., PETERSON, T.W. and SEINFELD, J.H., 1976, Dynamics of Aerosol Coagulation, J. AIChe $22 \mathrm{n}^{\circ} 05$. 